\title{
Implementing TCP Flow-Level Fairness Using 802.11e in a Multi-Radio Mesh Testbed
}

\author{
Venkataramana Badarla, David Malone, and Douglas J. Leith
}

\begin{abstract}
TCP is known to be subject to poor performance in multi-hop mesh networks. While most work has focused on interference and related issues, we note that cross-layer interactions can also induce considerable unfairness. In this paper, we propose a simple 802.11e scheme to mitigate these interactions and regulate TCP fairness in a flexible manner. The practical effectiveness of the approach is confirmed in a multihop, multi-radio testbed.
\end{abstract}

Index Terms-802.11e, CSMA/CA, TCP, test-bed.

\section{INTRODUCTION}

$\mathbf{T}$ HE problems faced by TCP in 802.11 multi-hop mesh wireless networks have been the subject of considerable interest in recent years. However, most of this work has focused on interference and radio-noise related issues. Recently, [1] noted that the MAC layer can lead to undesirable bandwidth allocations for voice traffic in wireless mesh networks. Similar unfairness problems exist with TCP traffic in these networks. This unfairness exists independently of lower layer interference issues and can interact badly with TCP's congestion control mechanism. While it is a known issue in single-hop WLANs (e.g. [2] and references therein), it has received little attention in a multi-hop context.

In this paper, we demonstrate that this is a significant issue for TCP in a multi-hop network and, moreover, using simple 802.11e settings we can substantially improve TCP performance. The effectiveness of the proposed scheme is confirmed using an experimental testbed equipped with multiradio relay stations built using commodity hardware. The approach creates a straightforward framework for implementing a wide range of fairness policies in multi-hop networks using commodity hardware.

We begin by highlighting two issues, both caused by 802.11's tendency to give the same number of transmission opportunities to each wireless station when the network becomes busy. The first problem is that TCP expects the return path for TCP ACKs to be uncongested. Congestion in the reverse path has long been known to be a problem for wired TCP, (e.g. [3]) and is also known to be an important problem for TCP in single-hop 802.11 WLANs (e.g. see [2] and references therein). This problem is also present whenever TCP flows are aggregated at a relay hop in a mesh network. This leads to queueing and loss of TCP ACKs and so to performance degradation due to disruption of TCP ACK clocking. The

Manuscript received December 11, 2007. The associate editor coordinating the review of this letter and approving it for publication was N. Nikolaou. This work was supported by Science Foundation Ireland grant IN3/03/I346.

The authors are with the Hamilton Institute, National University of Ireland, Maynooth, Co. Kildare, Ireland (e-mail: David.Malone@nuim.ie).

Digital Object Identifier 10.1109/LCOMM.2008.072083.

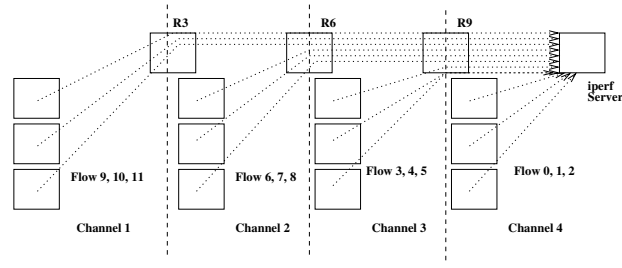

Fig. 1. Parking lot topology. Rn denotes a node relaying $n$ flows.
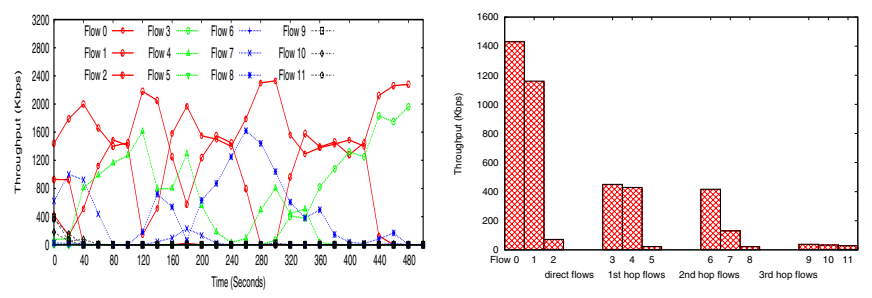

Fig. 2. TCP performance in 802.11 parking lot topology of Figure 1.

second problem is that at a relay hop a relay station, regardless of how many downstream stations/flows it is relaying packets for, will receive the same number of transmission opportunities as other contending stations. As a result, flows that travel through a relay station carrying many other flows may receive only a small share of the available bandwidth.

We illustrate the impact of these effects for the 4-hop parking lot topology shown in Figure 1. TCP flows travel from each station shown to the rightmost station. TCP ACKs are routed in the opposite direction. Each hop is on an orthogonal radio channel, and the relay stations are multi-radio, thus we avoid interference and hidden node problems and can focus on MAC/transport layer interactions. Figure 2 shows TCP goodput against time for 12 simultaneous TCP flows, one from each station. We see that the throughput is highly erratic and that flows travelling a smaller number of hops tend to starve flows travelling a larger number hops.

\section{802.11E Mesh Testbed SetuP}

We implemented the topologies shown in Figure 1 and Figure 5 using a testbed constructed from Soekris net 4801 stations with Atheros $802.11 \mathrm{a} / \mathrm{b} / \mathrm{g}$ miniPCI cards. The NICs used support 802.11e EDCF functionality which makes adjustable the MAC parameters AIFS, CWmin and TXOP. All stations run Linux 2.6.21.1 with a version of the MADWiFi driver customised to allow the priorisations described in this paper. Otherwise, tests were performed in infrastructure mode using standard 802.11a parameters and channels with PHY rate $6 \mathrm{Mbps}$. To implement dual-radio mesh relay points, we 

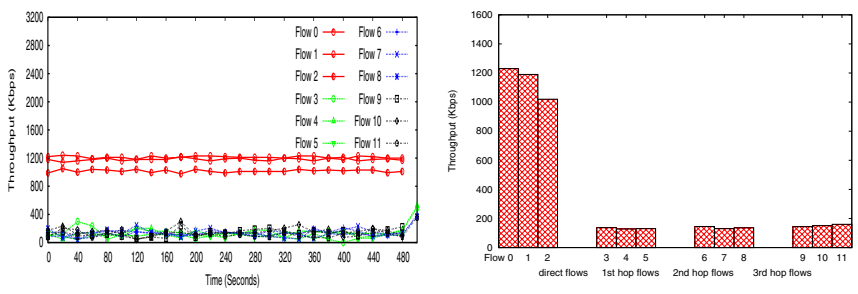

(a) Prioritised TCP ACKs
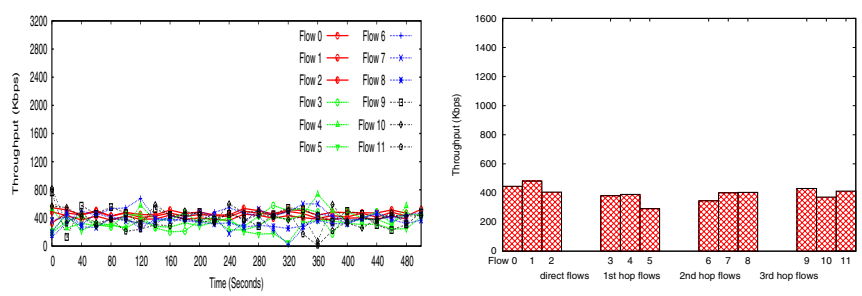

(b) Prioritised TCP ACKs and TXOP

Fig. 3. Improved 802.11e performance for parking lot topology.

joined two net $4801 \mathrm{~s}$ at $100 \mathrm{Mbps}$ with a cross-over cable to form a single logical mesh point. This avoided potential interference between network cards sharing the same PCI backplane. Routing in the network was statically configured. Iperf was used to generate TCP traffic and data was collected from both iperf and tcpdump. TCP flows used SACK enabled NewReno.

\section{PRIORITISING TCP ACKS}

First we address TCP ACK congestion. Similarly to [2], by increasing the AIFS of data packets by 4 slots and decreasing the CWmin of TCP ACKs to $2^{2}$ we can effectively give ACKs priority over data packets. Allowing TCP ACKs prioritised access to the wireless channel does not lead to the channel being flooded. Instead, it ensures that the volume of TCP ACKs is regulated by the transport layer rather than the MAC layer. In this way the volume of TCP ACKs will be matched to the volume of TCP data packets, thereby restoring forward/reverse path symmetry at the transport layer. To see the effect of this change, compare Figure 2 to Figure 3(a). We see that TCP's performance is now much more predictable, and even flows that travel many hops are not starved.

\section{Achieving Per Flow Fairness}

While prioritising TCP ACKs ensures more predictable TCP performance, it is evident from Figure 3(a) that a wide range of throughputs are achieved by the flows, with flows 0-2 significantly out-performing the other flows. We can understand this by recalling that the DCF enforces per-station fairness, i.e. each station contending for access gains roughly the same number of transmission opportunities. Per station fairness takes no account of the number of flows being relayed by a station. Hence, when we have a station relaying $n$ flows contending with a station relaying a single flow, each station is allocated a $1 / 2$ share of the channel capacity even though this means that the $n$ flows are each allocated a $1 / 2 n$ bandwidth share while the single flow gains a $1 / 2$ share. Over a number of cascaded hops, this type of behaviour can lead to the flow level unfairness potentially becoming large.

To address this unfairness, we consider how to achieve per flow rather than per station fairness. Here "flow" refers to any suitable administrative quantity; its precise definition is a policy decision for the network operator. For example, a flow could consist of all packets originating from one IP address, or group of IP addresses, rather than the usual source and destination address/port tuple.

We propose that TXOP, 802.11e's packet bursting mechanism, provides a straightforward fine-grained mechanism for controlling fairness. Let $n_{1}^{i}$ be the number of outgoing flows at a station on radio $i$ (stations may have multiple radios). To achieve a per-flow fair allocation of bandwidth, at each station we choose TXOP equal to $n_{1}^{i}$ for TCP traffic transmitted by radio $i$. That is, we transmit a number of packets equal to the number of flows carried.

To see the impact of this TXOP allocation, let $n^{i}$ be the number of stations contending for opportunities on channel $i$. Let $n_{j}^{i}$ be the TXOP used by station $j, j=1,2, . ., n$. The share of transmission opportunities allocated to station $j$ is then approximately $n_{j}^{i} / \sum_{j=1}^{n^{i}} n_{j}^{i}$. Since station $j$ transmits $n_{j}^{i}$ flows, the per flow share is $1 / \sum_{i=1}^{n^{i}} n_{j}^{i}$. In other words, transmissions are shared equally amongst the flows. Note that even if a station has a certain allocated value of TXOP time, if during a transmission opportunity a node has no packets to send then that transmission opportunity is ended. Thus, if the offered load at a station is too low to make full use of its allocated TXOP share, the excess is not lost but rather becomes available on a best effort basis for use by other stations in the network.

We demonstrate the impact of this TXOP allocation strategy in the parking lot topology of Figure 1, setting the 802.11e TXOP parameter to 3,6 and 9 packets for the left, middle and right relay stations. The impact on TCP flow throughput is shown in Figure 3(b). It can be seen that the per flow throughputs are now close to equal.

\section{A. Avoiding large packet bursts}

Increasing TXOP may result in long periods of uninterrupted transmission by one station, possibly adversely affecting delay sensitive traffic at other stations. An alternative to such single long transmissions is to use multiple shorter transmissions via a combination of TXOP and CWmin. E.g., halving TXOP at a station halves the burst size, but by also halving CWmin of the station we double its relative transmission chances, so that overall the station transmits the same number of packets as before. This mechanism achieves multiple shorter transmissions instead of a single long transmission. The number of contending stations and their traffic load places a lower constraint on CWmin to avoid excessive loss due to collisions. This can be off-set by increasing CWmin at other stations.

The mean MAC delay when using DCF at relaying nodes R9, R6 and R3 are $6.4 \mathrm{~ms}, 2.5 \mathrm{~ms}$ and $2.3 \mathrm{~ms}$, respectively. For TXOP the delays at these relaying nodes are $3.3 \mathrm{~ms}, 2.6 \mathrm{~ms}$ and $2.8 \mathrm{~ms}$; for $\mathrm{TXOP} / 2$ and $\mathrm{CWmin} / 2$, these delays are as $3.3 \mathrm{~ms}, 2.5 \mathrm{~ms}$, and $2.8 \mathrm{~ms}$. So, using TXOP actually improves 


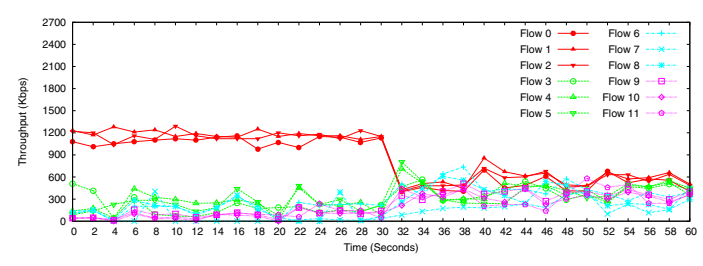

Fig. 4. Convergence when optimised parameters are applied.

the mean delay at R9, while having little impact at R6 and $\mathrm{R} 3$, where the network is less busy.

\section{B. Dynamic reconfiguration}

The number of flows in a network is typically not constant. It is usually straightforward for a station to determine the number of flows that its is transmitting. For example, if a flow is defined by its address/port tuple then inspection of the network interface queue allows direct measurement of the number of currently active flows. TXOP settings can be readily adjusted on the fly - potentially at every transmission. This is illustrated, in Figure 4 where TXOP at each station is initially at its default value of one (0-30s) and then TXOP adaptation to the number of flows is enabled (30-60s). We can see that following this major change there is a transient phase where the changes are applied from $30 \mathrm{~s}-32 \mathrm{~s}$, and by the $34 \mathrm{~s}$ the performance is close to what we expect from the new parameters.

\section{Other topologies}

Figure 5 shows an example of a hierarchical mesh topology. Here two flows come from the left hand side of the hierarchy and four from the right. Again, we consider the impact of prioritising the TCP ACKs and then setting TXOP to match the number of TCP flows relayed by a node. Figure 6 shows the performance of the stations with DCF alone, the network when we use the TCP ACK prioritisation scheme and TCP ACK prioritisation with TXOP. The results shown are an average of 10 runs and error bars show 95\% confidence intervals. Again, we see that TCP ACK prioritisation had reduced the variability of the results substantially. Further, by adapting TXOP, we have been able to create almost exact fairness between flows.

\section{Impact on throughput and network capacity}

The overall network throughput is also shown in Figure 6. In addition to achieving fairness among the flows, the proposed scheme yields a $\sim 15 \%$ increase in network capacity over DCF. This gain is associated both with the increased efficiency (reduced MAC overhead) due to the use of larger TXOP bursts and the positive impact of TCP ACK prioritisation on TCP performance. These benefits outweigh the cost of increasing AIFS for TCP data.

While the proposed scheme improve the fairness and capacity, it is subject to the usual difficulties due to misbehaving/selfish/uncooperative nodes. These could try to gain more access by using larger TXOP values than they are entitled. To counteract this, APs could police the transmissions of the

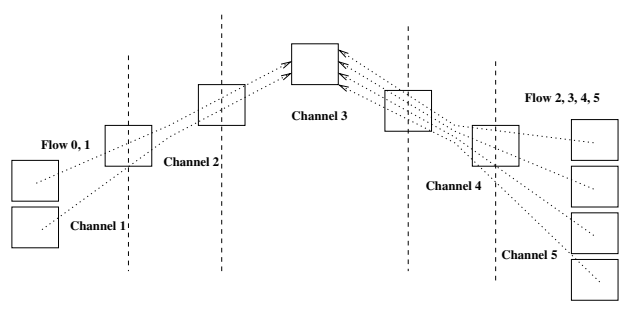

Fig. 5. Hierarchial topology.

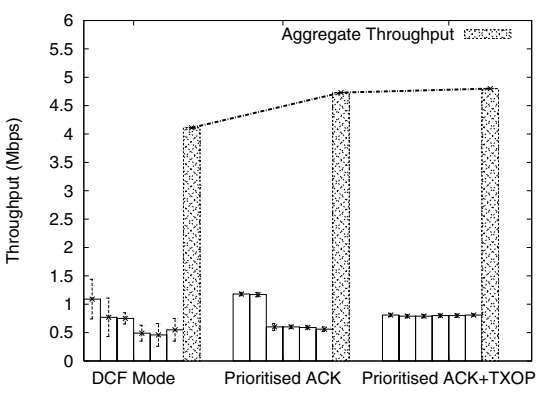

Fig. 6. Overall results from Hierarchial topology.

stations by observing TXOP lengths and comparing them to the number of active flows. Similarly, the APs could police ACKs sent per-flow to ensure the number is inline with the volume of TCP data transmitted. This would be in the spirit of existing systems for detecting greedy behaviour in 802.11 (e.g. [4]).

\section{Discussion}

We have demonstrated that the proposed scheme yields improved fairness and throughput, while being simple to implement on commodity hardware. These basic techniques can be readily extended in a number of directions. For example, differential prioritisation between TCP flows can be achieved by associating weight $w_{f}$ with flow $f$, selecting $\mathrm{TXOP}=\sum_{f=1}^{n_{j}} w_{f}$ and using a slightly modified queueing discipline at network interfaces. This $802.11 \mathrm{e}$ approach provides a simple and direct approach to distributed fair scheduling, avoiding complex message passing and custom scheduling schemes e.g. [5], [6].

\section{REFERENCES}

[1] K. Duffy, D. Leith, T. Li, and D. Malone, "Modelling 802.11 mesh networks," IEEE Commun. Lett., vol. 10, no. 8, pp. 635-637, 2006.

[2] D. Leith, P. Clifford, D. Malone, and A. Ng, "TCP fairness in 802.11e WLANs," IEEE Commun. Lett., vol. 9, no. 11, pp. 964-966, 2005.

[3] T. Lakshman, U. Madhow, and B. Suter, "Window-based error recovery and flow control with a slow acknowledgement channel: a study of TCP/IP performance," in Proc. INFOCOM, 1997.

[4] J.-P. H. M. Raya, I. Aad and A. E. Fawal, "DOMINO: Detecting MAC layer greedy behavior in IEEE 802.11 hotspots," IEEE Trans. Mobile Comput., vol. 5, no. 12, pp. 1691-1705, 2006.

[5] V. Kanodia, C. Li, A. Sabharwal, B. Sadeghi, and E. Knightly, "Distributed multi-hop scheduling and medium access with delay and throughput constraints," in Proc. ACM MOBICOM, 2001.

[6] M. Alam, M. Hamid, and C. Hong, "QoS-aware fair scheduling in multihop wireless ad hoc networks," in Proc. ICACT, 2006. 